\section{Compression in hypertensive leg ulcer (Martorell's ulcer)}

\section{Patricia Senet \\ Department of Vascular Dermatology, Tenon Hospital, Paris, France}

In 1945, Martorell, a Spanish cardiologist, described a peculiar form of leg ulcer that bears his name. He originally described patients with anterolateral lower leg ulcer(s), associated with arterial hypertension, and characterized by the presence of peripheral pulses, the absence of arterial calcifications, and of chronic venous insufficiency. These ulcers were related to arteriolosclerosis process involving the vessels of the dermis. Martorell's ulcer was then renamed as hypertensive leg ulcers (HLU) and more recently, the term arteriolosclerotic ulcer has been suggested.

HLU occur in long-standing hypertensive patients, usually women over 60 years old. Arterial hypertension is present in 95 to $100 \%$ of the cases, and diabetes has been reported in up to $40 \%$ of the patients. Notably, for 5 to $10 \%$ of patients, diabetes was reported as the unique cause. The aging of the population and the improvement of hypertension management seem to be responsible for a progressive drift towards older patients (around 75 yearsold) than before.

The original description of HLU and the subsequently reported cases showed that the clinical pattern and wound history were very similar from one series to another. HLU starts as a painful rapidly purpuric, necrotic and finally ulcerated lesion, of the antero-lateral side of the leg or on the posterior side of the ankle, including on the Achilles tendon, surrounded by a red purpuric margin spreading regularly and centrifugally. The second clinical characteristic of HLU is its extreme pain, more severe than would be expected for the size of the ulcer. A two phases evolution may be seen with the stabilization of one lesion, while another occur in another area or even on the other leg. Size of the ulcer is highly variable, but mostly between 2 and $100 \mathrm{~cm}^{2}$. Delay of healing is between 3 to 11 months, while it is shortened with skin grafting. According to the initial definition of HLU, the existence of significant peripheral arterial occlusive disease should be an excluding criteria for this diagnosis. However, it is not so clear as venous or arterial insufficiencies are far from being uncommon in the elderly. HLU occurs as a result of a micro-angiopathic process. The patients with hypertension disclose a high cutaneous vascular resistance despite normal ankle brachial pressure indices, due to narrowing or occlusion of the dermal arterioles. Besides, arterioles are not able to respond to the physiological dilatation reflex that occurs in response to diminution of the blood perfusion. Fully developed subcutaneous arteriosclerosis seems to be constant in HLU biopsies, and may have a very good diagnostic sensitivity, although its specificity is poor.

Differential diagnoses include calciphylaxia, cutaneous vasculitis, skin thrombosis (thrombophilia, anti-phospholipide syndrome, cryoglobulinemia, etc.), or other conditions such as pyoderma gangrenosum.

HLU management includes: i) pain control: opioids or their derivatives are often necessary. Combination with narcotic analgesics should be systematically associated (pregabalin or tricyclic antidepressive therapy) because of the neuropathic pain, which is a common component. The application of topical steroids on the purpuric active borders was reported to provide relief, and may be used only few days, in order to help controlling pain; ii) wound debridement and dressing: hydrogel, foam dressing or non-adherent silicone dressing. Debridement of fibrous and necrotic areas should be performed carefully, using local topical analgesia; iii) skin grafting: Grafting remains the most promising treatment strategy. Pinch or mesh skin grafts are used, with the same efficiency, leading to a complete healing between 4 to 6 weeks; iv) miscellaneous treatments. Various treatments have been tried without showing a beneficial effect on healing: topical recombinant human platelet derived growth factor, BB gel, heparine or coumadine, lumbar sympathectomy, hyperbaric oxygen therapy. Prostanoids may be promising; v) compression. Despite no study, use of venous compression is mentioned in HLU since many years. Venous compression is indicated before the graft, in presence of limb edema. As pain is extreme and constant, there is a decrease in the calf muscle pump because of a significant reduction of walking, leading to edema and to reduced skin perfusion. This may explained also why the pain is increased in patients presenting HLU when they are in standing position. With local care, venous compression participates to pain relief. Moreover, up to $40 \%$ of the patients have a venous insufficiency proved on venous duplex ultrasound
Correspondence: Patricia Senet, Department of Vascular Dermatology, Tenon Hospital, rue de la Chine, 75970 Paris cedex 20, France.

E-mail: patricia.senet@tnn.aphp.fr

This work is licensed under a Creative Commons Attribution 4.0 License (by-nc 4.0).

CCopyright P. Senet, 2016

Licensee PAGEPress, Italy

Veins and Lymphatics 2016; 5:5987

doi:10.4081/vl.2016.5987

examination. During and after cutaneous graft, venous compression is also used to reduce the limb edema, to enhance the take of the cutaneous graft and to maintain the graft on the wound. Compression device that are used in this indication are multisystem components (2 to 4 layers) with high stiffness to obtain a low resting pressure, and pressure fluctuations during walking. The level of compression depends on the tolerance and on the ankle brachial pressure index: $20-30 \mathrm{mmHg}$ may be enough but $40 \mathrm{mmHg}$ is generally well tolerated. Prevention relies on the correct management of hypertension and avoiding non-selective $\beta$-blockers, which will lower skin perfusion pressure. The patient should avoid traumatic incidents on the legs, protect them, and wear elastic compression stockings 20-30 $\mathrm{mmHg}$ to stimulate the blood velocity. ${ }^{1-4}$

\section{References}

1. Hafner J, Nobbe $\mathrm{S}$, Partsch $\mathrm{H}$, et al. Martorell hypertensive ischemic leg ulcer: a model of ischemic subcutaneous arteriolosclerosis. Arch Dermatol 2010;146:961-8.

2. Senet P, Beneton N, Debure C, et al. Hypertensive leg ulcers: epidemiological characteristics and prognostic factors for healing in a prospective cohort. Ann Dermatol Venereol 2012;139:346-9.

3. Senet P, Vicaut E, Beneton N, et al. Topical treatment of hypertensive leg ulcers with platelet-derived growth factor-BB: a randomized controlled trial. Arch Dermatol 2011;147:926-30.

4. Vuerstaek JD, Reeder SW, Henquet CJ, Neumann HA. Arteriolosclerotic ulcer of Martorell. J Eur Acad Dermatol Venereol 2010;24:867-74. 